\title{
OBSERVATIONS ON THE PASSAGE OF WEED'S PRUSSIAN BLUE MIXTURE ALONG THE AXIS CYLINDERS AND INTER-FIBRE FLUID OF NERVES
}

\author{
BY \\ E. J. FIELD \\ From the Department of Anatomy, University of Bristol
}

The idea that the axis cylinder is the path by which the causal agent of rabies reaches the central nervous system after peripheral inoculation is of considerable antiquity. Duboué (1879) is stated by Gamaleia (1887) to have urged axonal transmission to the complete exclusion of other pathways. In more recent times experimental support for this pathway has been forthcoming from Goodpasture and Teague (1923) working with herpes, Goodpasture (1925) working with rabies, and Fairbrother and Hurst (1930) working with poliomyelitis. More direct visual evidence was adduced by Perdrau (1937) who studied the passage of a number of "dyes" especially Weed's solution (a mixture of potassium ferrocyanide and iron ammonium citrate) along nerves both in situ and when excised. Using a weak cataphoresis current Perdrau found that this mixture could pass some 4 to $5 \mathrm{~cm}$. along the sciatic nerve in four hours. He also demonstrated the passage of the solution into the spinal cord where a selective staining of some anterior horn nuclei took place. Moreover he claimed that the dye permeated axis cylinders selectively, though staining of the connective tissue sheaths of the nerve also took place. With colloidal solutions, for example, Victoria blue, no trace of impregnation of axis cylinders was obtained. In a critical review of axonal transmission Doerr (1939) emphasizes this failure to impregnate the axis cylinder by colloidal solutions (more nearly related to virus particles than are true solutions) and also the staining of the connective tissue sheaths. Since Perdrau did not consider passage by diffusion in the interfibre fluid, though some of his findings would seem to be most readily explicable on this basis, the present work, largely a repetition of his, was undertaken.

\section{Material and Methods}

Four experiments were carried out on the sciatic nerve in situ in the rabbit anaesthetised with nembutal, and 11 further experiments on lengths of sciatic nerve excised in continuity with the lumbar cord. In three additional experiments rat sciatic nerve was used. In the first group the sciatic nerve was exposed at mid-thigh level, freed for about one centimetre, divided with a sharp razor blade, and the epineurium picked up with a fine oiled black silk suture on an eyeless needle. By this means the proximal end of the nerve was threaded for about $5 \mathrm{~mm}$. into a capillary glass tube suitably bent so that it could be readily fixed in situ when the posterior thigh muscles were brought together. Perdrau's injunction to obviate all kinking or compression of the nerve was found to be most important. A $10 \%$ mixture of sodium ferrocyanide and iron ammonium citrate made up in Ringer's solution was introduced by a needle into the open end of the glass tube so that the $5 \mathrm{~mm}$. of nerve within was bathed in the mixture. Considerable difficulty was experienced in preventing a " creep " of fluid between the nerve and the capillary tube. After some trials the tube was filled with fine sawdust kept saturated with the solution. The spinal cord was exposed through a small lumbar laminectomy, and a nonpolarisable (Ag-AgC1) electrode was placed in contact with its surface. Another was introduced into the open end of the capillary tube, this latter being connected up to the negative pole of a battery and the cord electrode to its positive pole. The voltage applied was adjusted so that a current of 0.3-0.5 milliamp flowed in the circuit. The cord contact was kept moist otherwise this current fell markedly. Experiments were continued for six to eight hours, more anaesthetic being given as required. At the end of that time the nerve was dissected out in continuity with the cord and fixed in $1 \% \mathrm{HCl}$ in $10 \%$ formol for 24 hours. In the series of experiments carried out on the excised nerve cataphoresis was employed in seven cases. In four of these the length of nerve attached to the cord was short-about $1.5 \mathrm{~cm}$.

Several further experiments were made to determine the permeability of the intact and damaged epineurium to prussian blue mixture. Lengths of nerve were excised between fine ligatures and in some cases a deliberate trauma was inflicted to breach epineurial continuity about the middle of the nerve segment. In others a short segment was cleaned of epineurium as far as possible under the microscope so that only a minimal 
connective tissue investment remained in this region. The pieces were immersed in the dye mixture for periods of four to six hours.

All specimens after fixation were embedded in paraffin, sectioned at $7-10 \mu$, and stained with carmalum.

\section{Observations}

Impregnation of axis cylinders was never so extensive as that observed by Perdrau (1937). Thus after six hours' cataphoresis there was staining of connective tissue sheaths for some $4 \mathrm{~cm}$. above the point of immersion of the nerve and coloration was not visible under the microscope above the level at which definite naked eye coloration ceased. Immediately above the immersed nerve segment there was diffuse staining of all the elements of the nerve. In the thin microscopic section the blue coloration of the epineurium, so readily visible to the naked eye in the gross specimen, was quickly lost. The diffuse blueness of the nerve fibres also faded quickly as one passed up the nerve and short sections of intensely stained nerve centred on the nodes of Ranvier stood out (Fig. 1a), the internodes becoming at first very faintly stained and then not noticeably coloured. Sometimes an annular effect was observed (Fig. 1b). In general, stained nodes were met with close up against the epineurium rather than in the depths of the nerve. The prussian blue seemed often to spread more readily in the circumferential part of the nerve (Fig. 2). Between the intensely staining nodes of Ranvier the axis cylinders remained apparently free from colour. From these appearances no unequivocal conclusions could be drawn about the selective passage of the prussian blue mixture up the axis cylinders from their severed ends. Indeed, the collar of dyestuff shown in Fig. 1b might equally be interpreted as resulting from the diffusion of prussian blue in the thin film of interfibre fluid with staining of axis cylinders at points where they are accessible to the fluid, i.e., the nodes of Ranvier. The fact that the nodes are stained higher up the nerve in its circumferential part supports this view, for the subepineurial space might be expected to allow more ready diffusion of dye (Brierley and Field, 1949, Fig. 6d). Perdrau (1937) remarked on the blue ring often to be seen at the nodes of Ranvier, and his description would seem to imply acceptance of the spread of the prussian blue other than along the axis cylinders, though he makes no mention of interfibre fluid. The system of capillary spaces within a nerve would seem to present the necessary conditions for the operation of powerful electrophysical forces which might result in surface streaming over nerve fibres. A phenomenon which offers certain points of analogy has been recorded by McFarlane (1940) who found that when a buffered suspension of vaccinia virus was placed in a Tiselius U-tube, the virus particles showed a streaming along the centre of the tube whilst the buffer moved in the opposite direction. In some parts of the present material appearances suggestive of diffusion along the neurilemmal surface were encountered (Fig. 3). Here fibre surfaces are stained by prussian blue which outlines well the nodes of Ranvier while the axis cylinders are only feebly coloured. Why nodes should stain so intensely and almost selectively when the concentration of prussian blue is low is not known. It may be that the interval between myelin segments accommodates interfibre fluid from which the prussian blue is thrown down on acidification. However, the nodes of Ranvier are known to possess special electrical properties (Tasaki and Mizuguchi, 1948) and it is possible that a high ferrocyanide ion concentration is held in their vicinity.

Perdrau's finding that impregnation of a piece of lumbar cord through an attached nerve root may lead to a marked staining of certain nuclei of anterior horn cells is easily confirmed (Fig. 4), though this could not be achieved if the length of nerve attached to the cord exceeded $1.5 \mathrm{~cm}$. On the other hand his finding that the nuclei of dorsaff root ganglion cells do not take up the mixture is subject to qualification, for no difficulty was experienced in achieving such impregnation provided that a short nerve root was used (Fig. 5). Perdrauk was able on rare occasions to trace a coloured axiso. cylinder from the surface of the cord up to the stained anterior horn cell. This has never been possible in the present material. Naturally the success of nuclear impregnation depends upon the concentration of prussian blue which reaches the neighbourhood of the nuclei. A low concentration may stain the nucleolus alone, but that a high concentration leads to the intense staining is shown in Fig. 4. It has been found that in an area containing several well stained anterior horn nuclei careful oil immersion examination reveals a sparse, fine, dust-like deposit of prussian blue, staining no element selectively. The appearances in the cord would appear to result therefore from the especial affinity of certain anterior horn nuclei and nucleoli for the prussian blue mixture which diffuses nonselectively throughout the cord substance.

\section{Discussion}

The main findings reported by Perdrau (1937) with regard to the passage of prussian blue mixture along nerves, especially when aided by cataphoresis, have been confirmed. While there can be no doubt that axis cylinders do take up the prussian blue 


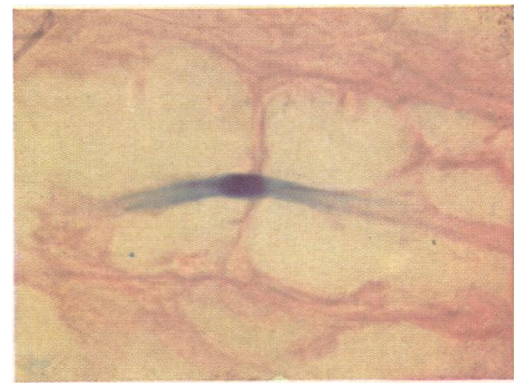

Fig. $1 a$

FIG. $1 b$
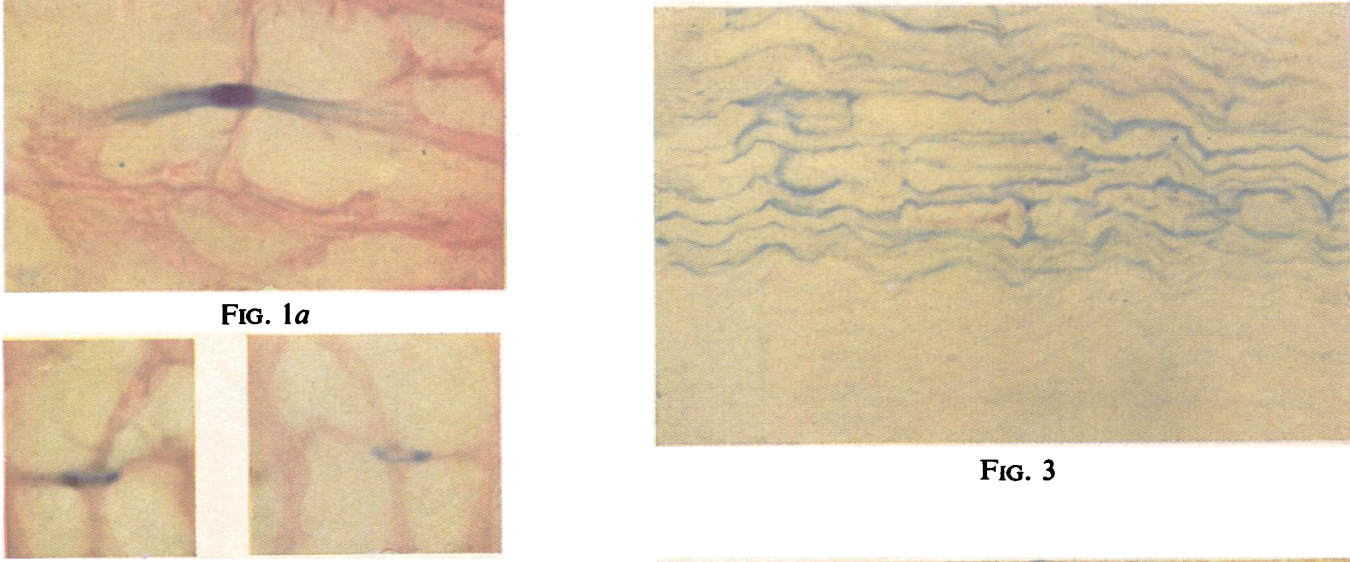

FiG. 3

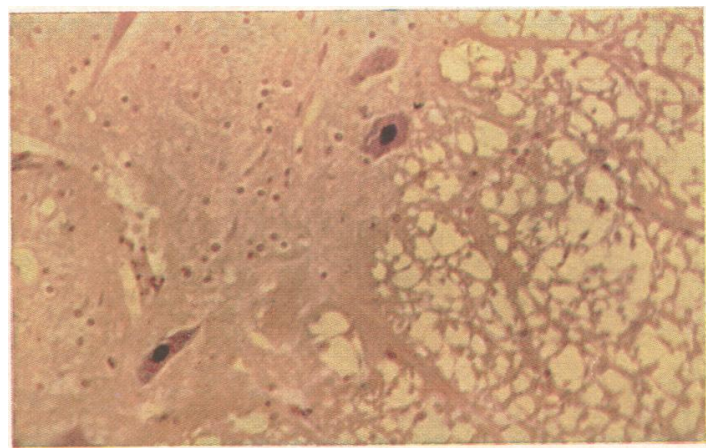

FIG. 4

FIG. 2

FIG. 1 a.-Deeply stained node of Ranvier in rabbit sciatic nerve about $1.5 \mathrm{~cm}$. above point of immersion in Weed's solution. $\times 1180$.

Fig. 1 b.- "Collar" of prussian blue around axis cylinder at node of Ranvier. About $2 \mathrm{~mm}$. above Fig. 1 a. $\times 1180$.

FIG. 2.-Sciatic nerve of rabbit about $1.5 \mathrm{~cm}$. above immersion level after six hours' cataphoresis. Note diffusion of the prussian blue in peripheral parts of the nerve. $\times 40$.

Fig. 3.-Same section as Fig. 2. Note prussian blue outlining the nerve fibres and dipping in between myelin segments at nodes of Ranvier, and the faintly coloured axis cylinders. $\times 300$

Fig. 4.-Transverse section of lumbar cord impregnated through an attached sciatic nerve root $(1.5 \mathrm{~cm}$.) by cataphoresis. Several nuclei have taken up prussian blue whilst neighbouring ones are quite uncoloured. $\times 120$.

Fig. 5.-Cell of lumbar dorsal root ganglion impregnated by cataphoresis. Note dense coloration of nucleus and particles of prussian blue on surface of nerve fibres in lower part of figure. $\times 650$.

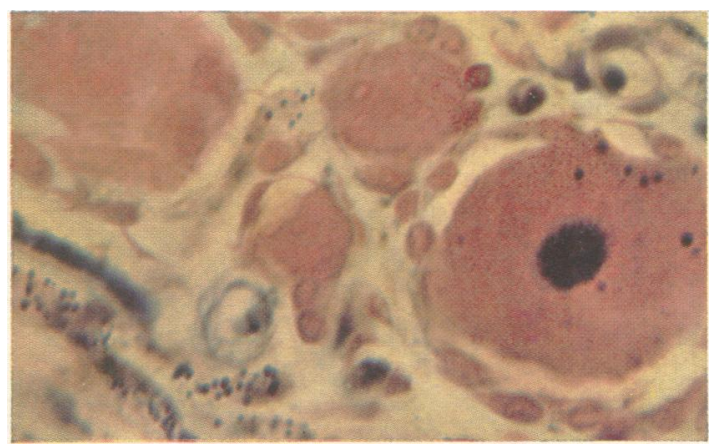

FIG. 5 
mixture readily the appearances do not seem to afford conclusive evidence that permeation takes place exclusively through the axis cylinder for any length. There is evidence that diffusion processes are active in the interfibre capillary spaces and in the subepineural region. In addition there is a surface creep in the epi- and peri-neurium. The results of immersing isolated nerve segment into prussian blue mixture suggest that a thick, intact epineurium will prevent staining of subjacent nerve fibres. However, if a segment of nerve is well stripped at one spot so that only a thin, investing connective tissue sheath remains, permeation readily takes place locally and spreads up and down the nerve. The connective tissue sheath would seem, therefore, to exert normally a mechanical protective action adequate to prevent the observed results being due to inward diffusion of the prussian blue mixture.

It is interesting to recall that Römer (1913) in considering the spread of intracerebrally inoculated virus suggested that it might spread most easily along the "lymph channels accompanying the neurone". From the context it is evident that the channels he had in mind are analogous to the interfibre capillary spaces along which prussian blue has been observed to spread in the present work. It is along these spaces too that Marinesco and Dragonesco (1923) suggest that the virus of herpes fibrilis may spread from the cornea to infect the brain. These spaces can be readily shown up by the intraneural injection of india ink (Brierley and Field, 1949) or herpes virus (Field, 1950). In this latter case columns of lymphocytes and cells showing nuclear fragmentation are to be found between individual nerve fibres. According to Weiss (1943) there is a definite centrifugal motion of the interfibre fluid which would make it a poor vehicle for centripetal transport of particulate matter in the nerve. This according to Weiss affords added, though indirect, support to the theory that such afferent transport, expecially of neurotropic viruses proceeds within rather than between nerve fibres. However, the existence of a centrifugal flow such as that envisaged by Weiss does not of necessity exclude centripetal diffusion of particulate matter (Field and Brierley, 1948). Moreover Young (1945) has brought forward evidence which suggests that under normal conditions there may be a slow centrifugal streaming of axoplasm, and this, together with the fact that de Renyi (1932) demonstrated the axis cylinder to be possessed of a jelly-like consistency, offers indirect evidence against the axis cylinder as an important conducting element for particulate matter. Indeed, Hurst (1936) has conceded that $\cdots$ the chief difficulty in the general acceptance of [axonal transmission] lies in the physical constitution of the nerve fibre".

Teale and Embleton (1920), although they believed that some soluble substances might diffuse up axis cylinders, concluded that the neural lymphatics and lymphatic spaces play an important part in the process. Orr and Rows (1916) also emphasized the "lymph stream in nerves" and their connective tissue coverings as important in the transport of toxins and bacteria to the central nervous system, and Ponomarew (1928) and Uljanow (1929) brought forward evidence of the centripetal passage of toxins and other soluble substances in the connective tissue spaces of nerves.

This brief and by no means exhaustive review is sufficient to show that the axis cylinder, the endoneurial fluid (contained in the interfibre or "lymph spaces" of the nerve), and the nerve sheaths have all been held either singly or in combination to be the essential avenue of invasion of the central nervous system through a peripheral nerve. The results described in the present work do no more than indicate that all three are possible in some measure under the given experimental conditions. Doerr's caution (1939) in translating such results to the problem of the neural transmission of viruses is well founded.

The nuclear impregnation in the spinal cord and dorsal root ganglia picking out certain cells to the exclusion of others nearby deserves further consideration. It is possible that affinity for prussian blue is cependent upon an electrical surface charge of the nucleus and the fact that this itself varies with different functional states of the nerve cell. It is an interesting speculation that the negative charge on the poliomyelitis virus particle may be the means whereby it is anchored in the first place to the anterior horn cell nucleus. If any valid analogy may be drawn from the findings with the negatively charged ferrocyanide ion complex, such a " fastening on "would be very patchy; this is precisely what is found in the anterior horn cell degeneration of poliomyelitis. Moreover, Russell (1947) has established the influence of exercise on the localization and severity of paralytic poliomyelitis ; that is, the influence of the physiological state of the nerve cell on its liability to attack by the virus. It would seem to be a reasonable speculation that exercise may be a factor affecting electrical charge on the anterior horn nucleus.

\section{Summary}

Diffusion of a prussian blue mixture may take place in the axis cylinder, interfibre spaces, and connective tissue sheaths of a nerve. 
No special conducting power of the axis cylinder was noted.

The nodes of Ranvier take up prussian blue and become intensely stained even when the concentration in the environment is below the threshold of visibility.

Certain nuclei of the anterior horn cells also have a high affinity for prussian blue, and the same has been found to be true of dorsal root ganglion cells.

The main views on neural transmission of crystalloids and viruses are indicated, and some speculations made on the significance of nuclear staining of the anterior horn cells.

I should like to express my gratitude to Professor J. M. Yoffey for his interest and encouragement in this work, and to Mr. K. Hunt and Miss M. Le Bechee for some of the histological preparations. For the colour photography I am indebted to Mr. J. E. Hancock and Mr. L. G. Cooper.

\section{REFERENCES}

Brierley, J. B., and Field, E. J. (1949). Journal of Neurology Neurosurgery and Psychiatry, 12, 86.

Doerr, R. (1939). "Handbuch der Virusforschung", ed. Doerr, R. and Hallauer, C., Vol. 2, p. 791. Vienna.
Duboué, H. (1879). “De la physiologie pathologique et du traitement rationnel de la rage." Paris. Cited by Gamaleia.

Fairbrother, R. W., and Hurst, E. W. (1930). J. Path. Bact., 33, 17.

Field, E. J. (1950). Unpublished results.

- , and Brierley, J. B. (1948). J. Anat., Lond., 82, 198.

Gamaleia, -. (1887). Ann. Inst. Pasteur, 1, 63.

Goodpasture, E. W. (1925). Amer. J. Path., 1, 547.

-, and Teague, O. (1923). J. med. Res., 44, 139.

Hurst, E. W. (1936). Brain, 59, 1.

McFarlane, A. S. (1940). Trans. Faraday Soc., 36, 257.

Marinesco, G., and Draganesco, S. (1923). Ann. Inst. Pasteur, 37, 753.

Orr, D., and Rows, R. G. (1916). Edinb. med. J., 17, 78. Perdrau, J. R. (1937). Brain, 60, 204.

Ponomarew, A. W. (1928). Z. ges. exp. Med., 61, 93.

Rényi, de G. S. (1932). In Cowdry, E. V. "Special Cytology," p. 1371.

Römer, P. H. (1913). " Epidemic Infantile Paralysis (Heine-Medin Disease)." English translation by Prentice, H. R. London.

Russell, W. R. (1947). Brit. med. J., 2, 1023.

Tasaki, I., and Mizuguchi, K. (1948). J. Neurophysiol., 11, 295.

Teale, F. H., and Embleton, D. (1920). J. R.A.M.C., $35,132$.

Uljanow, P. (1929). Z. ges. exp. Med., 64, 638.

Weiss, P. (1943). Anat. Rec., 86, 491.

Young, J. Z. (1945). In "Essays on Growth and Form ", edited by Clark, W. E. Le Gros, and Medawar P. B., p. 41. Oxford. 